\title{
The use of Microcontact Printing of Thiols to Evaluate Attachment of Xylella fastidiosa Under Distinct Conditions of Calcium Availability.
}

\author{
Breno Leite ${ }^{1}$, Rafal Dziedzic $^{2}$, L. F. Cruz ${ }^{3}$, A. L. Gillian-Daniel ${ }^{2}$, Charlie Nielsen $^{1}$ and L. De La Fuente ${ }^{3}$ \\ 1. JEOL USA, Inc., Peabody, MA, USA \\ 2. University of Wisconsin, MRSEC Education Group, Madison, WI, USA \\ 3. Auburn University, Department of Entomology \& Plant Pathology, Auburn, AL, USA \\ (bleite@jeol.com)
}

Plant Pathogenic bacteria attach to surfaces to form biofilms and effectively colonize the host plant. Xylem vessels internal surface attachment is the first step towards colonization during the infection of grapevines by Xylella fastidiosa [1], causative agent of Pierce's disease, an economically important factor for the California wine industry. Auburn University [2,3] is devoted to identify and test factors that are favorable to attachment and those that are not. The study of this xylem-limited pathogen is critical to establish how pathogenic cells reach massive growth inside vessels and eventually alter plant growth and/or cause death. Massive growth also means enormous production of virulence factors, such as toxins, and extended vessels blockage of normal flow of nutrients and water. The goal of this investigation is to test a methodology that can reduce the time of initial screening of chemical influencing factors and speed up experimental protocols. We accomplished these goals by: 1) monitoring biofilm formation under controlled conditions on glass surfaces coated with gold vs. gold surfaces coated with thiol (SH rich moieties), and 2) comparing obtained results with published results.

Layered assemblies were manufactured at the University of Wisconsin, which simulates surfaces that typically interact with pathogens. We used artificial gold surfaces coated with an octadecamethiol monolayer, that causes alterations in their hydrophobicity. The hydrophobicity changes because - $\mathrm{SH}$ groups are attracted to gold and leave behind molecule tails that are averse to water. The change to a negative due to $\mathrm{SH}$ groups ionization $\mathrm{H}$ (S- remains on the surface). Negative sulfur is attracted to positively charged gold. The technique is called Microcontact Printing of Thiols (http://education.mrsec.wisc.edu/294.htm). We tested a penny print (Figure 1A, B) and a designed pattern (not shown). Biofilm growth was tested by growing $X$. fastidiosa in PD2 growth media liquid cultures: control and supplemented media. Prepared slides were sterilized with ethanol inside the laminar flow hood and transferred to Falcon tubes containing PD2 [4] media and incubated for 5 days at $28^{\circ} \mathrm{C}$. After incubation slides were dried and fixed with osmium tetroxide. Treatments were: 1) Control PD2 medium $+X$. fastidiosa (inoculated media used as positive control); 2) PD2 $+2 \mathrm{mM} \mathrm{CaCl} 2+X$. fastidiosa (Complex media PD2 supplemented with calcium) and 3) PD2 $+1.5 \mathrm{mM}$ EGTA $+X$. fastidiosa (Complex media PD2 supplemented with the calcium chelator EGTA).

Scanning Electron Microscopy (JSM-6610 JEOL) was used to image surface growth. Images aimed to highlight differences between areas outside and inside the thiol printed areas. Biofilm and/or cell colonization was assessed by processing the data collected with the National Institutes of Health "ImageJ" software (http://imagej.nih.gov/ij/). The colonized area \% was correspondent to the area with bacterial cell forms/Total area analyzed. Data has confirmed that: a) X. fastidiosa cells attach preferentially to surfaces with higher degrees of hydrophobicity (Figure $1 \mathrm{~B}$ ); b) calcium $\left(\mathrm{CaCl}_{2}\right)$ significantly enhances cell attachment and aggregation (Figure $1 \mathrm{C}$ ) and c) EGTA reduces attachment. Results corroborate previously obtained data [2,3,4]; however this new method is less time consuming. 
Repeatability and standardization will likely be needed to introduce this method as a routine screening step in many labs devoted to test the influence of chemicals and other factors on colonization of many pathogens. Preliminary tests will be then refined for more complete and conclusive work. Data also allowed a better rationalization of how layers are assembled in vivo (Figure $1 \mathrm{D}$ ); We foresee the increase of data generation allowing: a) establishment of common basis for attachment of plant pathogens, b) help determine the foundation of the physics and chemistry involved in interactions of cells and their environment.

References:

[1] A.J. Simpson et al (2000). Nature 13 (406): 151-9.

[2] L.F. Cruz et. al. (2012). Appl. Environ. Microbiol. 78 (5): 1321-31.

[3] L.F. Cruz et. al.(2014). Appl Environ Microbiol, 12. pii: AEM.02153-14. [Epub ahead of print].

[4] B. Leite et al. (2004). F. of European Microbiology Society 230: 283-290.

[5] P.A. Cobine et. al. 81 (2013) PLoS ONE: e54936. doi:10.1371/journal.pone.0054936.

Authors acknowledge Dr. Maria L Ishida (FDACS) for critically reviewing the manuscript:
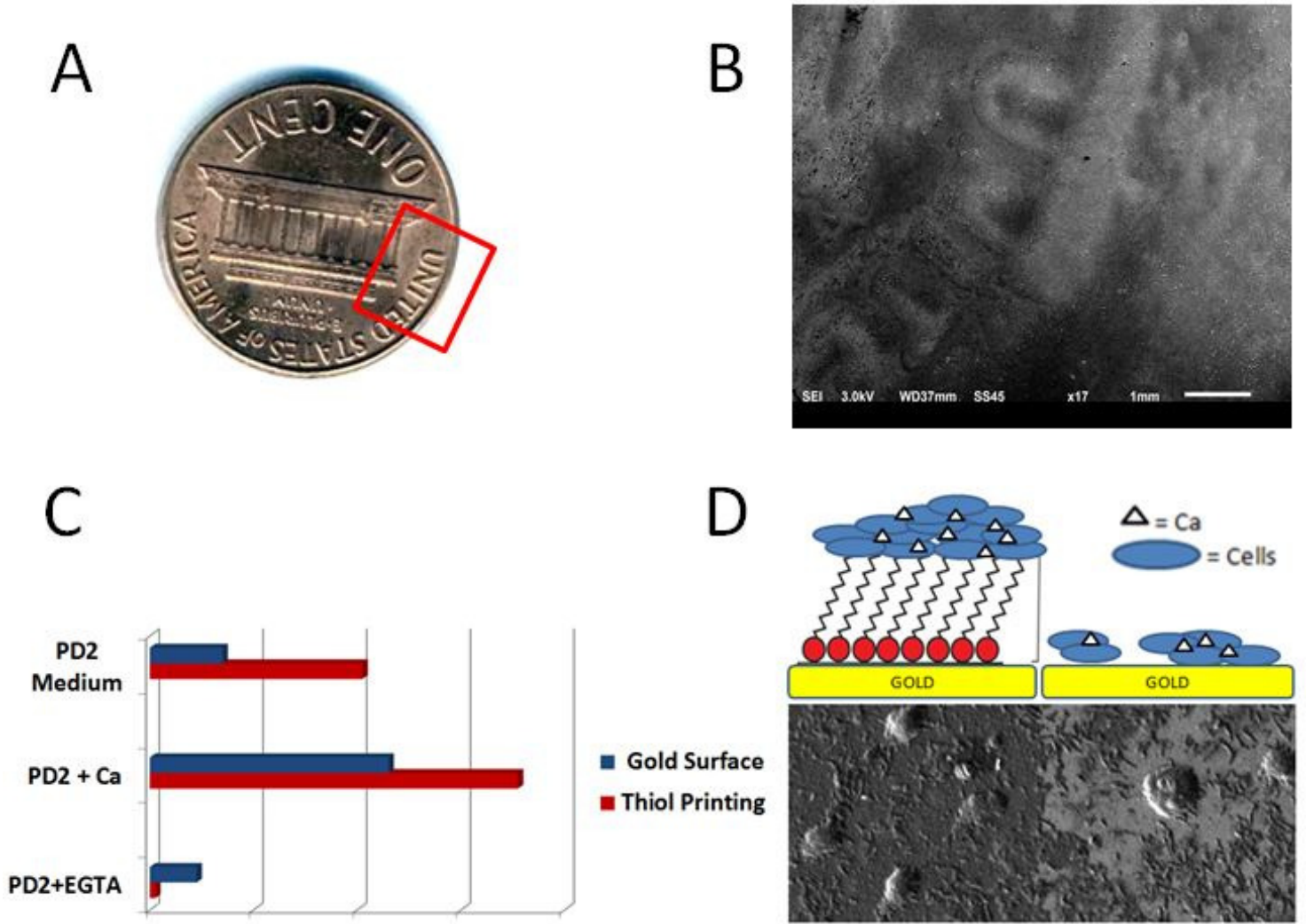

Figure 1. Region of a penny reproduced by thiol print on the right image (A); Vigorous $X$. fastidiosa on the thiol print penny areas (B); Area \% occupied by cells and biofilm (Image $\mathrm{J}$ software ) either on top of a gold surface or thiol printed areas. PD2 (control); CaCl2 supplied and in the presence of EGTA, a calcium chelator (C). Rationalization of why calcium mediation increases surface colonization (D). 